\title{
Vices et vertus du travail spécialisé
}

\author{
Jean-Louis Peaucelle
}

Gérer et Comprendre 2009 N $^{\circ} 97,28-38$

Résumé : Le travail spécialisé, nommé depuis Adam Smith « division du travail », offre des avantages et des inconvénients. Adam Smith mettait l'accent sur la productivité. Or il semble que se soit surtout la mécanisation qui augmente la productivité et, pour utiliser au maximum des machines onéreuses, on y spécialise un ou plusieurs opérateurs. Par ailleurs les tâches spécialisées sont plus rapides à apprendre. Cette organisation du travail permet d'intégrer rapidement une main d'œuvre non formée à la production. De plus, Charles Babbage a montré que, dans un processus avec une spécialisation, les écarts de salaires abaissent le prix de revient. Si on mesure la productivité non plus par la cadence, mais par la production utile, il faut tenir compte des temps morts liés à des déséquilibres des charges entre postes de travail, aux défaillances de la planification ou à l'aléa des arrivées du travail. Tous ces facteurs rendent efficace une polyvalence des opérateurs.

Mots clés : division du travail, spécialisation, polyvalence, Adam Smith, Charles Babbage, productivité, cadence, temps mort

Keywords : division of labour, specialization, skillfulness, Adam Smith, Charles Babbage, productivity, rate of work, slack period

\section{Introduction}

La « bonne » organisation du travail semble être celle où chaque personne, affectée à une machine, y répète la même tâche. Cette représentation est fondée d'une part sur la pratique des organisateurs, Taylor au premier rang d'entre eux, et d'autre part sur la théorie de Adam Smith sur la division du travail. Cet article a pour but de remettre en cause ce présupposé de l'excellence du travail spécialisé, dans toutes les situations. Cette synthèse s'appuie sur les nombreuses études, tant empiriques que théoriques, concernant le travail, celui d'hier comme celui d'aujourd'hui, dans les usines et en dehors des usines.

La spécialisation du travail ${ }^{1}$ est favorisée par deux aspects ; tout d'abord la mécanisation, source principale d'une haute productivité et en ensuite la brièveté de l'apprentissage avant le mise au travail. Pour ces deux raisons, en phase de croissance, la production de masse organise le travail en postes spécialisés.

Mais la productivité n'est pas le seul critère d'efficience. Pour que les prix de revient soient bas, avec le travail spécialisé, il faut aussi que les flux de travail arrivent sans à-coups. Dans une production irrégulière, la planification des tâches spécialisées laisse presque toujours des ressources inoccupées, même en univers certain. Or toute inoccupation de personnel ou de machines correspond à un coût. A l'inverse, la polyvalence crée des flexibilités utiles à chaque fois qu'on s'écarte des conditions optimales. II convient donc de distinguer les situations de travail en fonction de la variabilité des productions, quantitativement et qualitativement. Ceci permet de considérer comme un cas très particulier la production de masse, à la chaîne, en quantités constantes, avec des ouvriers spécialisés, sans formation antérieure. Dans ce cas particulier le travail est spécialisé, mais n'est pas l'archétype de tous les types de travaux productifs.

Par ailleurs, la spécialisation du travail suppose que les tâches puissent être analysées avant leur exécution par l'opérateur. Ce n'est pas toujours possible, notamment dans le travail en équipe et dans les situations d'opposition.

Ainsi le travail spécialisé n'est pas efficient dans tous les cas, et quand il l'est, c'est pour des raisons différentes de celles de la théorie de Adam Smith. Pour lui, la productivité s'accroît avec la division du travail. La pratique de Henri Ford réfute cette théorie. En 1914, en bon taylorien, il teste diverses organisations de sa chaîne de montage de voitures et il choisit celle ayant la meilleure productivité. Ce n'était pas celle où le travail était le plus divisé. Avec 177 ouvriers affectés à une tâche de 1 minute, le temps humain pour sortir une voiture était de 3 heures. Mais 44 ouvriers, travaillant chacun 2 minutes, montaient une voiture en 1,5 heure. La productivité était double sur cette chaîne où le travail était moins parcellisé. Cette organisation fut adoptée naturellement. ${ }^{2}$

${ }^{1}$ Adam Smith utilisait le terme de « division du travail », Taylor ou Ford jamais. En suivant leur exemple, on parle ici de "spécialisation ». L'expression « ouvrier spécialisé » est d'ailleurs employée dans ce sens par les patrons et les syndicats dans les conventions collectives. 
Le travail n'était donc pas le plus parcellisé mais il était cependant spécialisé par postes de travail, liés aux machines. Cet exemple confirme que la spécialisation dans les usines est liée à la mécanisation.

\section{La machine pour une haute productivité}

Dans le règne animal, l'homme se distingue par ses outils. II les choisit, les construit, les conserve pour une réutilisation. II accomplit ainsi ses tâches avec moins d'effort, notamment il produit mieux et plus vite.

En général, une production est possible de plusieurs manières, avec plusieurs outillages. Parmi ces possibles, est privilégié celui qui fournit le meilleur prix de revient. Un nouvel outil est adopté s'il permet une productivité humaine plus élevée, s'il n'est pas cher, s'il ne tombe pas souvent en panne, si sa durée de vie est assez longue, s'il est d'usage simple après une formation rapide, si sa capacité de production correspond aux quantités à fournir sur le marché. L'arc est préféré au propulseur pour tuer les animaux, puis le fusil devient un meilleur outil de la chasse ou de la guerre.

Sans cesse, l'homme invente de nouveaux outils, de nouvelles machines. Les automates construits par Heron à Alexandrie au premier siècle APJC ou par Vaucanson au XVIIle siècle à Paris ont un but ludique. Ils restent dans les théâtres et dans les salons.

L'industrie adopte des machines quand elles abaissent le prix de revient. Comme les nouvelles machines sont chères, en général, ce critère d'adoption exige que le coût de main d'oeuvre soit plus bas et donc, à salaire égal, que la productivité soit plus forte. Pour cette raison, parmi les machines, celles qui sont sélectionnées par les industriels augmentent la quantité produite par heure de travail.

A un moment donné, peuvent coexister deux processus de fabrication, l'un avec des outils simples et une faible productivité, pour des quantités réduites, l'autre avec des machines complexes et une forte productivité, pour de grandes quantités. Par exemple, on recoud un bouton à la main avec une aiguille, mais la couturière emploie une machine à coudre et, dans les grands ateliers de confection, les machines sont encore plus perfectionnées. L'avantage de la machine dépend des flux de travail.

Si les machines sont peu onéreuses, il est possible de doter chaque ouvrier d'un outillage complet qui est faiblement utilisé. L'ouvrier effectue alors l'ensemble des tâches, en changeant d'outil. C'est la situation de l'artisan polyvalent, par exemple le plombier réparateur à domicile.

Si les machines sont onéreuses, on les fait fonctionner au maximum. Le coût fixe est d'autant plus faible pour chaque unité produite, que les machines sont utilisées continûment. On leur affecte de manière permanente les ouvriers chargés de les faire fonctionner. La spécialisation aux postes de travail a pour but d'utiliser à plein les machines chères. La spécialisation du travail dépend de la technologie. II n'y a donc aucun arbitraire organisationnel à un découpage des postes de travail dans le processus global. C'est la technologie qui dicte la répartition des tâches. C'est aussi elle qui augmente la productivité. Les deux effets sont liés à la même cause, l'usage d'une machine.

L'outillage est cause de la croissance de la productivité et de la spécialisation, contrairement à la théorie de Adam Smith pour qui la division du travail était la cause première d'une productivité plus forte. La mécanisation en était la conséquence.

"The division of labour, however, so far as it can be introduced, occasions, in every art, a proportionable increase of the productive powers of labour [...]

"This great increase of the quantity of work which, in consequence of the division of labour, the same number of people are capable of performing, is owing to three different circumstances; first to the increase of dexterity in every particular workman; secondly, to the saving of the time which is commonly lost in passing from one species of work to another; and lastly, to the invention of a great number of machines which facilitate and abridge labour, and enable one man to do the work of many." ${ }^{3}$

Adam Smith fondait ses réflexions sur la fabrication des épingles telle que la décrivaient les encyclopédistes français. Chacune des opérations de l'épinglerie correspondait à un outil différent, billot, bobille, engin, cisaille, meules, calibres de longueur, rouet, ciseaux, cuiller, entêtoir, chaudron, chaudière, plat à vanner, frottoire, quarteron. L'apparente division du travail était une spécialisation par outil. Les 18 opérations étaient les descriptions des gestes pour utiliser autant d'outils et un même ouvrier pouvait successivement passer d'un outil à un autre. II y avait donc une polyvalence des ouvriers. La distinction des opérations venait de l'outillage et la répartition du travail était très différente

${ }^{2}$ David Hounshell, 1984, From the American system to mass production, 1800-1932, The development of manufacturing technology in the United States, The John Hopkins University Press.

${ }^{3}$ Adam Smith, 1776, The Wealth of nations, L1, Ch1. 
dans un gros atelier et un petit. Or il y avait plusieurs centaines d'ateliers faisant travailler de 2 à 20 personnes, avec le même outillage. La répartition du travail était donc variable, parce que l'outillage n'était pas onéreux. Le prix de revient était le même. II n'y avait pas d'avantages particuliers pour les gros ateliers capables de spécialiser un ouvrier sur une seule opération ${ }^{4}$.

Ainsi, la fabrication des épingles confirme que l'outillage dicte le mode opératoire mais pas la répartition du travail. Les épingliers avaient la même productivité qu'ils aient été spécialisés par opération ou non, parce qu'ils avaient les mêmes outils. Cette réalité industrielle n'était pas du tout conforme à la théorie que Smith voulait illustrer par cet exemple. D'ailleurs au XIXe siècle, a été inventée la machine à faire les épingles exécutant toutes les opérations antérieures. Le travail a été recomposé, ou même s'est transformé en une surveillance de la machine pour l'alimenter en fil métallique. Cet exemple montre que les machines les plus productives ne sont pas toujours celles qui divisent le processus productif.

Lorsque des automatismes assurent complètement la production, le travail humain de surveillance, dépannage, entretien, conduite de processus, etc. n'est pas organisé comme celui des ouvriers spécialisés par poste avec des gestes répétitifs.

Dans les grands ateliers, la mécanisation induit une spécialisation du travail. De plus cela permet de faire travailler des personnes n'exigeant pas de hauts salaires.

\section{Mettre au travail des ouvriers sans compétence}

Au Moyen Âge, le travail était réalisé dans les villes par les artisans organisés en corporations. La compétence professionnelle était au cœur du fonctionnement de ces « communautés d'arts et métiers ». Les enfants étaient mis en apprentissage, pendant 5 ou 10 ans, sous l'autorité du maître de l'atelier, rémunéré spécifiquement par les parents. Cette formation se terminait par le « chef d'œuvre », examen pratique montrant la connaissance de tous les aspects du métier.

A cause de cet apprentissage long, la capacité de travail ne se modifiait que lentement. Comment faire quand la demande devenait brusquement très forte ? II fallait mettre au travail des personnes sans les former longuement.

Pour faire travailler rapidement des personnes non formées, on doit concevoir des tâches simples, avec un outillage spécifique, facile à manier. Alors, on forme très vite les personnes à exécuter ces tâches. On recrute les ouvriers les moins qualifiés, ceux qui acceptent les salaires les plus bas.

Ces outils, spécifiques à une partie du processus productif, s'opposent aux outils à usage multiple. Ils contiennent les réglages spécifiques à l'opération. Un outil générique permettrait de faire le même travail, mais il faudrait apprendre à le manœuvrer exactement, à le régler. Par exemple, pour ajuster les têtes des d'épingles, antérieurement, l'artisan épinglier frappait avec un marteau. Une machine spécialisée a été construite, l'entêtoir. Une masselotte coulissant verticalement sur des tiges de guidage jouait le rôle du marteau. Là où il fallait savoir ajuster l'orientation et la vitesse du marteau, il a suffit de déclencher la chute de la masselotte. Vitesse et orientation étaient constantes. L'entêtoir était une machine spécifique et il demandait peu de compétence. Il était manipulé par des femmes payées deux fois moins que les hommes.

A la lecture des textes de l'Encyclopédie, Charles Babbage a remarqué que la spécialisation offrait un avantage en termes de prix de revient. Certaines opérations étaient effectuées par des femmes moins payées. D'autres exigeaient une compétence plus élevée, celle d'un professionnel mieux payé.

"The higher the skill required of the workman in any one process of a manufacture, and the smaller the time during which it is employed, so much the greater will be the advantage of separating that process from the rest, and devoting one person's attention entirely to it". ${ }^{5}$

« Si l'on faisait exécuter l'ensemble d'un processus technique par un seul travailleur, ce dernier devait posséder le niveau de qualification requis par l'opération la plus difficile, et l'ensemble de la tâche devait être rémunéré en fonction de ce niveau. En revanche la division du travail, [...] diminuait les coûts de l'entrepreneur, qui ne payait qu'au prix minimal les tâches non qualifiées ou peu qualifiées

${ }^{4}$ Toutes les références à la fabrication des épingles sont tirées de Jean-Louis Peaucelle, 2007, Adam Smith et la division du travail, la naissance d'une idée fausse, L'Harmattan.

${ }^{5}$ Charles Babbage, 1832, On the economy of machinery and manufactures. London: Knight. $p$. 186. Réédité en 1993. London : Routledge. 
confiées à des travailleurs sans qualification et qui employait des travailleurs qualifiés seulement dans les tâches pour lesquels ils étaient nécessaires $»^{\cdot 6}$

Les machines spécialisées sont simples d'usage pour un néophyte qui apprend rapidement à s'en servir. Elles interdisent la plus part des erreurs de manipulation. L'apprentissage est aussi réduit quand le geste opératoire se répète, quand la durée du cycle est courte. Ce temps opératoire descend jusqu'à 3 secondes par exemple pour fixer la tête de l'épingle. Les novices deviennent vite compétents sur l'opération qu'on leur réserve après qu'on leur ait montré plusieurs fois au ralenti, puis en les faisant faire. En quelques minutes, ils ont le temps de répéter le geste de nombreuses fois.

Adam Smith confond l'apprentissage rapide avec la vitesse d'exécution. Celle-ci ne peut pas augmenter au-delà de la vitesse maximale des gestes, vitesse déterminée par la physiologie humaine, la contraction des muscles et leur contrôle. En revanche, plus l'ensemble des gestes est de durée réduite, plus vite on apprend à les exécuter.

Former rapidement de nouveaux ouvriers offre d'abord l'avantage de les rémunérer au plus bas niveau du marché du travail. De plus, on peut répondre à une croissance rapide de la demande. Les nouvelles capacités de production sont rapidement mises en place en spécialisant le travail, avec une main d'oeuvre nouvellement embauchée. Quand l'activité se stabilise, on conserve généralement la même organisation en postes spécialisés. Quand l'activité diminue, la spécialisation devient un inconvénient majeur. Les ouvriers qui ont opéré sans cesse sur la même activité ne savent plus faire que cela. Ils ont beaucoup de mal à se reconvertir, d'autant plus qu'on leur propose seulement des postes exigeant une polyvalence, donc une formation et une compétence qu'on ne leur avait pas demandée auparavant.

Cette production de masse, à la chaîne, avec des ouvriers spécialisés, est au coeur du développement économique des XIXe et XXe siècles. La productivité progresse régulièrement. L'entreprise fait des bénéfices, baisse son prix de vente et paie de bons salaires. Clients, patrons et salariés y trouvent avantage. C'est le compromis fordiste. Cependant les dirigeants d'entreprise raisonnent en général sur le prix de revient qui dépend aussi du niveau des salaires. Ils délocalisent la production dans des pays à bas niveau de salaire.

Le travail par postes spécialisés est observable dans l'histoire industrielle, à chaque fois qu'on a été confronté au même problème de mise au travail d'une main d'œuvre sans formation professionnelle. En voici des exemples anciens.

Dans une économie principalement agricole, le travail suit les saisons. Lors des récoltes, il faut rentrer le plus vite possible le blé arrivé à maturité. Avant les moissonneuses batteuses, cela exigeait une très grande quantité de main d'œuvre. On y faisait travailler toute personne disponible. Ces moissonneurs occasionnels constituaient une main d'œuvre non formée. On leur réservait les tâches simples et répétitives de la mise en bottes par exemple. Une partie de la production y était donc spécialisée, bien que cela se passe en dehors des ateliers.

Une spécialisation par opération a commencé à exister avant la révolution industrielle. Dans les sociétés riches, les miséreux posent toujours un problème. Aux Pays-Bas, dès le XVle siècle, on les a secourus et on les a fait travailler, quand ils étaient valides, dans les " ateliers pour pauvres ${ }^{7}$. Ils y venaient irrégulièrement et on les mettait à des tâches réduites, faciles à apprendre, pour des salaires très faibles. Ces ouvriers quittaient ces ateliers dès qu'ils le pouvaient. Le processus de production des épingles décrit par Adam Smith a été inventé en Hollande dans ces ateliers pour pauvres.

Au moment où Frederick Taylor s'intéressa à l'organisation, l'industrie américaine employait de nombreux travailleurs immigrés d'Europe Centrale, sans aucune formation industrielle. Elle était donc confrontée au problème décrit ci-dessus et la même solution a été mise en œuvre.

\section{Le travail spécialisé en opposition à la polyvalence}

Le poste de travail taylorien est défini par la machine ou l'outil utilisé à cette étape du processus de production. Le mode opératoire, c'est à dire les gestes de l'ouvrier, est défini après expérimentation comme la manière de faire qui permet d'aller le plus vite. Ce rythme devient la cadence " normale » ou « temps alloué », de quelques secondes à quelques minutes. ${ }^{8}$

${ }^{6}$ Patrick Verley, 1994, Entreprises et entrepreneurs du XVIIle siècle au début du XIXe siècle. Paris : Hachette. pp. 12-13.

${ }^{7}$ Les ateliers pour pauvres ont aussi existé en Grande Bretagne à partir du règne de Elisabeth I. 
Ceci concerne le poste de travail. Taylor innove vraiment en préconisant 8 chefs fonctionnels. Quatre d'entre eux s'occupent de la planification du travail : les agents de lancement qui indiquent l'ordre des travaux à accomplir, le spécialiste de l'usinage des métaux qui prépare la vitesse de coupe pour chaque travail, le spécialiste de l'étude des mouvements et le spécialiste du calcul des temps opératoires.

Le plan ne suffit pas, il faut le respecter. Le chef hiérarchique fait respecter la discipline. Mais Taylor lui adjoint 4 contremaîtres fonctionnels supplémentaires : les contrôleurs qui vérifient que l'ouvrier comprend le mode opératoire, les chefs d'équipe qui s'assurent que la machine-outil fonctionne à la bonne vitesse (cadence) avec les bons outils, les contremaîtres d'entretien qui donnent les directives sur le nettoyage et le réglage des machines afin d'éviter les pannes, les employés des temps qui recueillent les productions réalisées et calculent les payes. Ainsi l'exécution des injonctions est vérifiée. Le travail se réalise comme prévu.

Sans cette planification et ce contrôle, la spécialisation aux postes de travail serait insuffisante pour assurer une haute productivité.

Par opposition au travail taylorien, un travail polyvalent est celui où l'ouvrier passe d'une machine à une autre, en un cycle beaucoup plus long, de 10 minutes ou de plusieurs heures. L'ouvrier polyvalent accomplit plusieurs tâches successives qui auraient pu être confiées à des opérateurs spécialisés. La rotation entre postes est un début de polyvalence. Un ouvrier qui sait travailler sur plusieurs machines peut chaque jour être affecté à un poste différent. Pour la motivation des salariés, Herzberg préconise ce travail élargi. Il prône aussi le travail enrichi où l'opérateur exécute lui-même une partie des tâches des chefs fonctionnels, il participe à la planification, il règle sa machine, il la nettoie et l'entretient, il trouve les bons gestes pour tenir la cadence, il vérifie ce qu'il réalise et signale lui-même sa production journalière. ${ }^{9}$

Selon la manière dont le travail est éloigné de l'organisation taylorienne, la polyvalence est plus ou moins complète. Plus elle est poussée, moins il est facile de décrire le travail, le prescrire et le contrôler.

Le salaire est un point fondamental dans la production. Son mode de calcul est un moyen pousser les ouvriers à produire. Si les tâches sont répétitives, on compte les quantités produites, et le salaire en est déduit. Ce salaire est considéré comme incitatif. Mais la difficulté est de fixer le tarif. Toujours, on a procédé à l'envers. Sachant la cadence normale et le salaire visé pour l'ouvrier, on en a déduit la formule de calcul. Le travail spécialisé a été un moyen de conserver dans les usines la rémunération aux pièces qui existait depuis très longtemps. Les épingliers du XVIIIe siècle étaient payés aux pièces.

Cependant, quand le rythme de travail ne dépend pas de l'ardeur de l'ouvrier, il est inutile de compter sa production individuelle. Dans la chaîne de Ford, comme dans les abattoirs de Chicago, les ouvriers sont payés $5 \$$ par jour, parce que la vitesse de la chaîne ne dépend pas d'eux ${ }^{10}$. De même, si un ouvrier manque de matières pour travailler, son temps de non-travail doit être rémunéré et on revient à des salaires fixes.

Si un ouvrier tourne entre plusieurs postes payés au rendement, il voit vite sur lequel il gagne plus ou se fatigue moins. II demande alors qu'on l'y spécialise ${ }^{11}$. Le salaire aux pièces peut être un frein à la polyvalence. Les organisations polyvalentes payent en général des salaires en fonction du temps de travail.

L'ouvrier spécialisé est réputé tenir des cadences plus élevées que le polyvalent. Mais il faut que l'organisation des flux de matières lui fournisse continuellement du travail. C'est la question de la planification et des temps inoccupés.

\footnotetext{
${ }^{8}$ Frederick Taylor, 1912, Principes d'organisation scientifique des usines, traduction de The principles of scientific management, 1911. Frederick Taylor, 1913, La direction des ateliers, traduction de Shop management, 1903.

${ }^{9}$ Frederick Herzberg, Bernard Mausner, Barbara Bloch Snyderman, 1959, The motivation to work, John Wiley.

${ }^{10}$ Jean-Louis Peaucelle, 2003, « Du dépeçage à l'assemblage, l'invention du travail à la chaîne à Chicago et à Detroit », Gérer et Comprendre, $\mathrm{N}^{\circ} 73,75-88$.

${ }^{11}$ Corinne Gaudart, 2003, "La baisse de la polyvalence avec l'âge : question de vieillissement, d'expérience, de génération?", Pistes, Vol. 5 No. 2, Décembre
} 


\section{Le planning et les capacités de production inemployées}

Considérons d'abord le cas où on connaît tous les éléments de la production avant de produire. Toutes les prévisions se réalisent sans écart. Le produit à réaliser est connu. Le producteur sait quelles quantités produire. II connaît les opérations nécessaires avec leur durée, le salaire des opérateurs, les outils ou les machines avec leurs coûts et leurs caractéristiques techniques. Ayant ces informations prévisionnelles sans erreur, il sait comparer à l'avance ces diverses organisations de la production. On se rapproche de cette situation en clôturant le lieu de production, l'usine ou le chantier. Les échanges avec l'extérieur sont contrôlés. Les incertitudes sur la production sont limitées.

Dans cet univers certain, les modes de production sont bien connus, selon les volumes : la production à la chaîne, en petites séries, à l'unité ou par projet. On a déjà parlé de la production à la chaîne, pour de grandes quantités. Quand les quantités sont réduites, la planification laisse des personnes partiellement inemployées, si elles sont strictement spécialisées.

Quand les quantités à produire sont plus faibles, chaque usine a une production différenciée, à I'unité ou en petites séries. On planifie l'ordonnancement de ces petites séries. II existe toujours un goulet d'étranglement formé par la machine pleinement employée ${ }^{12}$. Les autres postes de travail, s'ils sont spécialisés, ne sont pas occupés à temps plein. Le prix de revient en est obéré.

Alors que la situation est totalement prévisible, les opérateurs ne sont pas tous occupés continûment, à cause des contraintes de la planification. Les temps de non travail obèrent le prix de revient (ouvriers payés à ne rien faire, machines à l'arrêt) et font baisser la production journalière

De même dans un projet où tout est connu, il existe un chemin critique où les opérateurs sont totalement occupés. Pour les autres tâches, il existe des marges temporelles, c'est à dire que les personnes qui y seraient spécialisées ne sont pas complètement occupées (PERT) ${ }^{13}$. Cela génèrerait un coût inutile. Si elles sont polyvalentes, on les affecte temporairement à d'autres tâches.

Ces modèles classiques de planification de la production supposent une totale spécialisation. Si les ouvriers ont une certaine polyvalence, les temps morts liés à la planification peuvent être occupés à d'autres tâches, entretien, nettoyage, formation, préparation des travaux ultérieurs etc. Avec la polyvalence, on retrouve des marges de manoeuvres dans la planification pour éviter les moments de non travail. On améliore la capacité de production, la productivité et les prix de revient ${ }^{14}$.

La compétence multiple des personnes, dans une organisation avec postes spécialisés, facilite la construction des plannings ${ }^{15}$. Pour cette raison, il est souhaité dans l'Education nationale que les enseignants des Collèges soient bivalents, qu'ils enseignent deux matières.

Ainsi le travail spécialisé s'applique seulement à la production de masse, à la chaîne. La polyvalence existe pour les petites productions, pour des raisons d'efficience. Elle est encore plus recommandée en univers incertain.

\section{Le travail en univers incertain}

La totale prévisibilité de la production est bien sûr un cas limite. Il existe des écarts entre la réalité et les prévisions. Beaucoup d'observations sociologiques montrent que, sans que la direction s'en aperçoive, les opérateurs utilisent leur intelligence pour faire face à ces écarts, difficultés spécifiques, écarts sur les durées, écarts sur la nature des tâches, oubli de tâches, etc. Pour d'autres imprévus fortement visibles : absentéisme, panne, demande plus forte ou plus faible, etc. les gestionnaires recommencent la planification. C'est plus facile s'il y a une certaine polyvalence. Ainsi,

${ }^{12}$ Jeff Cox, Eliahu Goldratt, 1984, The goal: excellence in manufacturing. Corton on Hudson, NY: North River press. Traduction Le but : l'excellence en production. 1986. Paris : AFNOR.

${ }^{13} \mathrm{~N}$. Stilian. 1962, PERT: a new management planning and control ,technique, American Management Association.

${ }^{14}$ Konstantinos Chatzis, 1999, L'autonomie dans les organisations : quoi de neuf ?, Paris : l'Harmattan

${ }^{15}$ Nicolas Hatzfeld, 2000, "La polyvalence enjeu multiple dans l'organisation du travail et la relation salariale", La lettre du Gerpisa, $\mathrm{n}^{\circ}$ 139, février, 5-8. Armelle Gorgeu, 1998, Organisation du travail et gestion de la main-d'oeuvre dans la filière automobile, Paris : la Documentation française 
les petites erreurs de prévision sont absorbées par cette polyvalence, reconnue ou non. Le travail totalement spécialisé est une situation limite.

Si la demande varie beaucoup quantitativement, il faut adapter les capacités de production. Par exemple, on met deux équipes dans les mêmes ateliers, pour doubler la production. Au contraire, on réduit la production en affectant un même opérateur à plusieurs machines (cellular manufacturing ${ }^{16}$ ). Cet opérateur est donc polyvalent. L'usine est flexible.

La situation devient tout à fait différente quand on considère une demande aléatoire. C'est le cas des services de garde à l'hôpital, des pompiers, de la police, des équipes de surveillance des automatismes, des équipes commerciales dans un magasin, etc. La demande arrive de manière non prévue, comme les clients dans un restaurant où ils veulent manger vite.

On approche cette incertitude de la demande par les lois probabilistes. Dans un centre d'appels on ne sait pas exactement quand les clients téléphonent. On en rend compte assez bien avec des modèles combinant une saisonnalité et un aléa. On est capable alors de mettre en relation la qualité de service (les attentes des appels) et le coût (le nombre de téléopérateurs) selon les divers moments, moments où on prévoit un nombre moyen d'appels. Dans cet univers aléatoire, on optimise l'occupation des personnels et le temps d'attente des clients. On a ainsi la production d'un service dans des délais brefs avec une bonne productivité des agents. On concilie ainsi deux critères, productivité et délai de service ${ }^{17}$.

Tout travail en contact avec l'extérieur possède ces caractéristiques et c'est la raison pour laquelle Hammer recommande la polyvalence des agents. Polyvalents, ils traitent l'ensemble d'un dossier de bout en bout. "To have one person perform all the steps in a process" ${ }^{18}$. Cette approche de l'organisation, le BPR (Business Process Reengineering), traite de la même manière le travail des bureaux et celui des ateliers. Dans les bureaux aussi des agents spécialisés effectuent une tâche limitée et transmettent les dossiers à une autre personne. Par exemple, une personne calcule un total et une autre le vérifie. Une personne traite les aspects juridiques d'un appel d'offres, une autre règle les aspects techniques, une troisième calcule le prix de revient prévisionnel, une dernière décide du prix demandé dans la proposition. Hammer conseille de faire faire toutes les opérations par une même personne, et de la former à cela.

La rotation entre les postes est aussi une bonne organisation en milieu administratif. Par exemple, dans une université, le service d'inscription travaille de manière intense de juin à octobre. Le reste du temps il n'a pas de tâches à accomplir. Mais les agents de ce service sont spécialisés. Même s'ils font appel à des aides ponctuelles lors de la période d'inscriptions, ils se refusent à effectuer des tâches en dehors de leur domaine de compétence, même quand ils n'ont plus d'inscriptions à faire. Leur productivité ne dépend pas du temps élémentaire pour enregistrer un étudiant mais principalement de leur non occupation en dehors des périodes de pointe. Dans toutes les procédures périodiques, l'organisation spécialisée a une faible productivité à cause des périodes creuses. II faut régulièrement réaffecter les personnes en fonction des charges de travail, les rendre polyvalents.

Il existe des cas où on spécialise les opérateurs avec un coût très élevé, pour réduire les délais. Lors des compétions de formule 1 , une dizaine de mécaniciens attendent la voiture afin de ravitailler et changer les roues en moins de 10 secondes. Pendant le reste de la course, ils attendent. Leur productivité globale est faible pour une intervention rapide au moment opportun. De même le chauffeur du ministre consacre son temps essentiellement à attendre la fin des réunions de son patron. Le but est que celui-ci n'attende jamais. Ces services spécialisés coûtent cher.

La polyvalence est la plus efficace dans le couple de critères coût et délai. La raison en est simple. Supposons qu'on ait spécialisé les agents selon un critère quelconque, zone géographique ou type de problème, il peut se produire un moment où les clients saturent le poste d'accueil spécialisé qui leur est réservé alors qu'un poste de travail ayant une autre spécialisation est inoccupé à ce moment précis, faute de clients. Avec un traitement non spécialisé, les agents qui seraient inoccupés traitent le cas des clients qui auraient attendu. Pour le même coût, c'est à dire les mêmes effectifs, les attentes sont réduites. Face à une demande imprévisible, la polyvalence est une source d'efficience du travail du point de vue coût et délais.

La polyvalence offre ainsi des avantages économiques d'équilibrage des charges de travail. Mais elle est limitée par la durée des formations.

\footnotetext{
${ }^{16}$ Nanua Singh, Divakar Rajamani, 1996, Cellular manufacturing systems, London : Chapman \& Hall

${ }^{17}$ Patrice Flichy, 2002, Les centres d'appel. Paris : Hermès

${ }^{18}$ Michael Hammer, 1990, "Reengineering work : don't automate, obliterate", Harvard Business Review, July-August, 104-114.
} 


\section{La polyvalence limitée par la formation}

Est-ce que le travail spécialisé est le même que celui où il y a des spécialistes, des médecins par exemple ? Le médecin généraliste traite les maladies fréquentes. Les patients atteints de maladies plus rares sont confiés à des "spécialistes". La raison est celle de la durée de la formation. Un médecin généraliste suit 9 années d'études, le spécialiste 10 ans. Un médecin qui suivrait les 40 spécialités aurait des études cinq fois plus longues. La durée de la formation ne doit pas être trop longue afin que la durée de la vie professionnelle ne soit pas trop courte. Le traducteur qui voudrait connaître toutes les langues passerait sa vie à les apprendre sans avoir le temps d'exercer son métier.

Chaque métier est défini par l'acquisition des compétences : talents innés, formation initiale, formation permanente et entretien par la pratique. Un professionnel dont la compétence est trop large ne tarde pas à se spécialiser sur le genre de cas qu'il a eu à traiter en début de carrière. S'il veut acquérir d'autres compétences, il doit réinvestir dans une formation, théorique ou sur le tas. Cet effort limite l'étendue des zones de compétences.

La spécialisation par métier dépend aussi de la conservation des aptitudes. L'habileté dans le métier se conserve si on exerce ce métier, si on suit les évolutions de ses pratiques. La spécialisation aide à conserver ces aptitudes parce qu'elles sont sans cesse mises en oeuvre. L'ouvrier polyvalent qui n'a jamais eu l'occasion de traiter un cas rare est démuni quand il se présente. II a perdu sa compétence par manque d'exercice. Conserver sa compétence nécessite de l'exercer et oblige à se limiter à un certains cas.

Tous les travailleurs intellectuels, avocats, professions libérales, notaires, ingénieurs conseil, cadres, enseignants, chercheurs etc. ont ainsi une spécialisation plus précise que ne l'indique le nom de leur métier. Mais ils accomplissent des tâches variées et conservent toutes les capacités d'organisation de leur propre travail, méthode, outillage, rythme, calendrier de travail, prise de rendezvous, contrôle. Ils sont totalement polyvalents. Malgré la proximité des mots, leur travail n'est pas organisé comme celui des ouvriers spécialisés sur leur poste de travail. Leur spécialisation ne vient pas d'une machine particulière auxquels ils seraient affectés mais au type de problèmes qu'ils ont I'habitude de traiter.

Le choix d'un individu vers un métier ou un autre dépend de ses aptitudes propres. Le talent conduit vers une spécialité. Ceci est tout à fait indépendant de la race humaine. Par exemple chez les abeilles, celles qui sont moins sensibles aux chocs deviennent des gardiennes et les butineuses sont celles qui ont la plus forte appétence au glucose. ${ }^{19}$

Ainsi il existe des spécialités, découpées en fonction de la durée des formations, mais les tâches quotidiennes y sont polyvalentes, tant dans le travail industriel que dans le travail de bureau. On n'a certainement pas considéré toutes les situations de travail, mais se dessine l'idée de la généralité de la polyvalence. Le travail d'équipe et celui d'opposition, de concurrence, sont des situations de travail peu étudiées. La polyvalence y est largement développée.

\section{Le travail coopératif et le travail agonique}

L'organisation du travail a un but non explicite de fixer la coopération entre travailleurs. Le poste de travail se situe dans un processus et son rythme est contraint par celui des postes en amont et en aval. Le plan de production, les dossiers, la hiérarchie, les règles, les objectifs fixés pour chacun, sont autant de mécanismes de coordination. Henry Mintzberg distingue un mécanisme moins formel, l'« ajustement mutuel ${ }^{20}$. Les opérateurs se parlent librement et cela est utile pour qu'ils coordonnent leur travail. Curieusement, ce mécanisme est antérieur à l'industrialisation et il perdure dans les situations modernes les plus complexes. Or parfois cet ajustement mutuel, non prévisible et impossible à prescrire, fait partie intégrante du travail à accomplir.

a) le travail fondamentalement relationnel.

${ }^{19}$ Edith Roussel, J. Carcaud, J-C Sandoz, Martin Giurfa, "Reappraising Social Insect Behavior through Aversive Responsiveness and Learning". PLoS ONE, 4(1): e4197, 14 janvier 2009.

${ }^{20}$ Henry Mintzberg, 1979, The structuring of organizations: a synthesis of the research. Englewood Cliffs: Prentice Hall. Traduction : Structure et dynamique des organisations, Paris : Editions d'organisation, 1982. 
Dans certains métiers, les échanges constituent une grande part du travail. Le vendeur attend la parole de son client exprimant sa commande. II la provoque par son propre discours. Certes ce discours est un peu normalisé par les argumentaires de vente, mais il reste fondamentalement adaptable à chaque situation de vente. De même, dans les métiers d'aide à la personne, la parole échangée avec le malade, l'impotent, la personne handicapée, est un élément du service rendu. Même si les actes sont prescrits par le médecin, ils sont adaptés à chaque situation concrète. La polyvalence est nécessaire pour bien exercer ces métiers relationnels.

b) le travail à résultat collectif.

Dans certaines situations de travail, l'effort de chacun n'a de sens que quand il est complété par celui des autres. Par exemple dans un projet de conception d'un avion ou d'une voiture, la qualité du produit final exige une intense coopération avec les autres concepteurs. Ces échanges peuvent être formalisés par les ordres des chefs de projets, par des logiciels de CAO, par des procédures qualité, par des révisions périodiques. Mais il subsiste toujours des échanges imprévisibles et nécessaires. Construire la coopération est une des tâches du projet. Même si chacun a un métier particulier spécifique dans le projet, la polyvalence est la règle pour tenir compte des situations imprévues que crée la communication.

c) le travail ayant pour but de transformer le travailleur.

La formation a pour but que l'étudiant acquière des connaissances et des compétences. Le sportif s'entraîne pour améliorer ses performances ou l'amateur pour développer ses muscles et conserver la forme. Ces actions sont souvent un travail non rémunéré, travail parfois très dur, exclusif de toute autre activité. Le candidat au concours étudie plus de 80 heures par semaine pendant une ou plusieurs années. Le thésard consacre tout son temps à mener sa recherche et rédiger son mémoire. Le soldat nouvellement incorporé suit un entraînement dur pour s'aguerrir, s'habituer aux situations de combat. L'athlète dépense toute son énergie en de longues heures d'entraînement.

Dans ces activités, les conditions de travail sont mauvaises : intensité de l'effort, risque d'accident et d'échec élevé, pénibilité, faible rémunération, stress, hiérarchie autoritaire, concurrence entre pairs, etc. On peut y constater un absentéisme, des abandons (démissions), des suicides, des dépressions, une fatigue, un mal être, des comportements violents, une addiction (alcool, médicaments etc.). Cependant ces travaux sont tout à fait acceptés, avec une forte motivation, parce qu'ils sont temporaires et qu'ils conduisent à des travaux plus confortables.

L'entraînement n'est pas une activité spécialisée, même s'il demande de « répéter ». On y anticipe un ensemble de situations possibles ultérieurement. Le « programme » est varié et on passe sans cesse à d'autres acquisitions. Au contraire, la formation centrée sur le futur poste de travail des ouvriers spécialisés est très rapide et reproduit seulement les conditions du travail réel, travail spécialisé sur une machine.

La formation est un travail coopératif avec les enseignants qui conduisent la transformation de l'étudiant et la contrôlent au travers des examens, concours, tests, etc. Le résultat de la formation a besoin d'une reconnaissance. L'étudiant est ainsi obligé de savoir ce que les enseignants attendent de lui et de vérifier que ses efforts vont bien dans cette direction. De plus, la coopération entre étudiants est un moyen puissant d'émulation et d'approfondissement des savoirs et des compétences.

d) l'activité agonique.

Le travailleur, individuel ou collectif, s'oppose à un adversaire. C'est un jeu ou une concurrence, un match sportif ou la conquête d'un marché. Par exemple dans le jeu "papier-caillou-ciseau », gagne celui qui détecte les régularités du comportement de son adversaire. A l'inverse de la coopération où on exécute les souhaits du partenaire, dans la situation d'opposition, on anticipe les actions de l'adversaire et on y pare.

Les entreprises tentent de connaître les orientations de la recherche et les résultats de leurs concurrents. Les prix de revient, les plans stratégiques, les produits nouveaux sont autant d'informations conservées secrètes le plus longtemps possible. Ce secret est un élément de leur efficacité. Cette anti-coopération s'appuie sur les moyens d'information économique, sur l'espionnage, sur la désinformation.

Les sportifs sont dans cette situation comme au tennis ou au football. Le jeu est autant celui d'imposer ses combinaisons que d'obstruer les tentatives des adversaires. Pour y réussir, les équipes de haut niveau répètent des combinaisons défensives contre les manoeuvres connues des adversaires. Et ils mettent au point des combinaisons offensives nouvelles. Quand les adversaires ont 
bien assimilé les parades, il devient inutile de les utiliser. Cette situation de jeu d'opposition est structurée en deux moments, le match proprement dit, avec son résultat, et l'entraînement, avec un grand nombre d'exercices répétés.

Le joueur, même s'il occupe une place particulière sur le terrain, est largement polyvalent pour réagir librement aux conditions spécifiques de chaque phase de jeu.

Ces situations de travail non abordées classiquement par les organisateurs montrent que la spécialisation y est proscrite comme un moyen sûr de ne pas atteindre l'objectif.

\section{Conclusion}

Traditionnellement, une conception « humaniste » de l'industrie s'est opposée à l'organisation du travail spécialisé. Le travail répétitif lasse et fatigue. Les cadences et les postures imposées usent le corps. Pour améliorer les conditions de travail et motiver les opérateurs il faut une plus grande variété de tâches. La polyvalence est alors préconisée pour améliorer la qualité du travail sous ses différents aspects : ambiance et harcèlement, santé et accidents, salaire et congés, horaires, localisation, sécurité d'emploi, etc. Ces revendications humanistes ont eu des succès limités mais elles ont aidé à promouvoir la polyvalence alors même que celle-ci se justifie par le seul critère d'efficience économique.

Le travail spécialisé reste meilleur à chaque fois qu'il faut servir des machines onéreuses dans un flux de production régulier. C'est la situation de la production de masse avec le travail à la chaîne, situation particulière qui n'est pas le parangon de tout travail. Parce que les ouvriers y travaillent continûment, le travail spécialisé sur chaque machine offre une bonne productivité, mais dans ce cas seulement d'une production en série régulière. On y emploie des personnes sans formation et les salaires sont bas. La production de masse y a regroupé un grand nombre de travailleurs. Les automatismes et les robots y ont limité les effectifs dans les pays développés mais les productions délocalisées adoptent cette organisation.

Pour les autres productions, l'organisation polyvalente est surtout plus efficiente parce qu'elle minimise les temps de non-travail, elle permet un meilleur engagement des personnes, elle facilite la planification et la réaction aux aléas. Par exemple dans les bureaux, les flux de travail sont rarement à maîtrisés. La spécialisation y génère des attentes et des périodes de non-occupation. Elle conduit à des coûts inutiles.

La polyvalence n'est jamais totale. Elle est limitée par l'ampleur de la formation nécessaire. Apprendre à traiter tous les cas, y compris les cas rares, serait un investissement inutile. La cohérence des métiers et la durée des formations induit une spécialisation des personnes, comme les médecins sont spécialisés sur certaines pathologies.

La polyvalence dans l'organisation du travail ne signifie nullement que tous exercent le même métier. Chacun se spécialise en fonction de ses dons. Certains métiers exigent des aptitudes que ne possède pas l'ensemble de la population. Ceux qui les possèdent peuvent s'y spécialiser, ce sont les sportifs, les artistes, les chercheurs etc. L'inégalité des dons naturels crée des différences qui provoquent une spécialisation, par métier au minimum.

Si donc, aujourd'hui comme jadis, la polyvalence est l'organisation du travail adaptée à la plus part des situations de production, pourquoi donc avons-nous conservé le travail spécialisé comme référence de la « bonne organisation ». Le « nous » qui est interrogé ici est formé de toutes les personnes, ingénieurs, économistes, gestionnaires, sociologues, organisateurs, qui réfléchissent sur le travail.

Je voudrais suggérer une hypothèse, celle de la facilité intellectuelle. II est aisé de représenter l'activité répétitive d'une personne devant une machine accomplissant sans cesse le même geste, au même rythme. Décrire un travail polyvalent, non prévisible, s'inventant en se faisant, est beaucoup plus délicat. Les commodités de la description poussent à supposer que le travail est spécialisé. On a l'impression de mieux le comprendre. II s'agit d'un modèle insuffisant, d'une approximation, comme les « gaz parfaits » sont une première approche du comportement des gaz en fonction de la température et de la pression.

Pour illustrer cette idée, le cas de la description du chargement du fusil est intéressant. Les instructeurs militaires de Louis XIV identifiaient « 36 mouvements pour charger par le canon un mousquet » et « 23 pour le fusil au XVIle siècle ${ }^{21}$. La description de ce travail s'appuyait sur une décomposition du processus, mais le même soldat exécutait toutes les opérations puisque l'outil était

${ }^{21}$ Patrick Mortal, 2007, Les armuriers de l'Etat, Villeneuve d'Ascq : Septentrion, pages 21 et 22 
unique. On ne mettait pas un soldat à chaque opération. Dans ce cas, la méthode analytique ne faisait pas croire à un travail spécialisé, mais le procédé a été repris pour d'autres métiers.

Les processus artisanaux décrits dans l'Encyclopédie mettaient en scène quantité d'outils spécialisés. La mise en œuvre de chaque outil, dénommée opération, était décrite séparément et il était facile aux auteurs, comme aux lecteurs, de supposer une spécialisation des ouvriers, même si cela n'était pas abordé explicitement. Cette description conduisait sans le vouloir à se représenter le travail comme spécialisé, avec une répétition des gestes par la même personne. Avec des machines différentes, on imagine facilement une spécialisation du travail. Quand Taylor veut prescrire le travail et le contrôler, son paradigme est aussi de spécialiser les ouvriers et de leur imposer une vitesse.

En décrivant le travail de manière analytique, on privilégie l'idée d'une spécialisation et progressivement celle-ci devient le cadre intellectuel de perception du réel. On invente alors des théories pour justifier cette vision et on prône leur application. C'est un curieux exemple d'influence du regard des intellectuels sur l'activité elle-même. 